\title{
Commentary Strained in Planning Your Mouse Background? Using the HPA Stress Axis as a Biological Readout for Backcrossing Strategies
}

\author{
Jennifer C Chan', Amanda B Houghton' and Tracy L Bale*,I \\ 'Department of Biomedical Sciences, School of Veterinary Medicine and Perelman School of Medicine, University of Pennsylvania, Philadelphia, \\ PA, USA
}

Neuropsychopharmacology (2017) 42, 1749-175I; doi:I0.1038/npp.2017.66; published online 19 April 2017

The use of transgenic mouse lines over the last several decades has been indispensible for modeling human conditions and probing mechanisms underlying disease. Importantly, selecting the correct mouse background strain can be difficult and is, no doubt, critical to experimental outcomes, interpretation of results, and reproducibility. In the fields of stress and neuropsychiatric disease research, strain-dependent differences in treatment sensitivity, neuroanatomical development, stress and physiological responses, and performance on behavioral tests have directed laboratories toward using preferred mouse strains for reliable, robust results.

In the last 25 years, transgenic mice have paved the way toward a greater understanding of genes involved in disease pathology. However, when examining the Jackson Laboratories mouse inventory, the chances of finding a transgenic mouse on your preferred background strain are unlikely. In fact, the standard transgenic mouse-production pipeline of injecting embryonic stem cells (ES) with the incorporated transgenic modification into blastocysts typically uses 129derived ES cells and C57BL/6 blastocysts (Ledermann, 2000). The resulting successful chimeras are then backcrossed on a C57BL/6 background to determine transgene transmission. As agouti coat color is dominant over black, this cross allows penetrance determination. For convenience and to save time, labs then often continue breeding the mice to a C57BL/6 background, thus resulting in the extensive usage of this strain of mice for neurobehavioral outcomes. Numerous studies have corroborated the usefulness of the C57BL/6 mouse in neuroscience research as this strain excels in learning-dependent tasks (Holmes et al, 2002), shows clear preferences for sucrose and alcohol (Pothion et al, 2004; Yoneyama et al, 2008), gains weight in diet-induced obesity models (West et al, 1992), exhibits high levels of immobility in the forced swim test (Lucki et al, 2001), has high sensitivity to pain (Mogil et al, 1999), and responds well to

\footnotetext{
*Correspondence: Dr TL Bale, Neuroscience Center SOVM, University of Pennsylvania, 380S. University Ave, 4IOF Hill Pavilion, Philadelphia, PA 19|04, USA, Tel: +215-898-1369, Fax: +2I5-573-5187,

E-mail: tbale@vet.upenn.edu

Received 22 February 2017; revised 14 March 2017; accepted 16 March 2017; accepted article preview online 31 March 2017
}

antidepressant treatment compared to other inbred strains (Lucki et al, 2001). In comparison, substrains of the 129 background display significant difficulty with hippocampaldependent learning tasks and fear extinction (Hefner et al, 2008; Camp et al, 2012), and another popular inbred strain, $\mathrm{FVB} / \mathrm{N}$, also has problems with learning tasks and prominent visual impairments (Brown and Wong, 2007). Moreover, the C57BL/6 mouse was the first rodent to have its genome completely sequenced, again adding to its popularity amongst researchers and the disproportionately available literature and resources for this strain (Waterston et al, 2002).

For many labs, however, the C57BL/6 mouse is not advantageous. In 2005, Ducottet and Belzung reported that, among 8 inbred mouse strains tested, C57BL/6 mice were relatively resistant to mild chronic variable stress, rendering this strain less relevant for studies examining the role of stress experiences in the etiology of neuropsychiatric disorders (Ducottet and Belzung, 2005). Strain differences in baseline stress responsiveness and anxiety-like behaviors are likely critical components of strain disparities in stress susceptibility (Homanics et al, 1999; Mozhui et al, 2010). In contrast to C57BL/6 mice, BALB/c and 129 mouse strains produce significantly greater levels of corticosterone in response to an acute stress (eg, restraint) (Camp et al, 2012; Anisman and Matheson, 2005). These two strains also exhibit more anxiety-like behaviors in the elevated plus maze, light-dark box, and open field tests, and show greater susceptibility to chronic stress (Homanics et al, 1999; Anisman and Matheson, 2005). Therefore, the 129 and $\mathrm{BALB} / \mathrm{c}$ strains are more likely to provide face validity in studies modeling susceptibility to stress experiences as an increased risk for neuropsychiatric-related disorders than C57BL/6 mice (Anisman and Matheson, 2005; Ducottet and Belzung, 2005). Importantly, the use of specific inbred mouse strains based on behavioral and physiological characteristics to model disease does not parallel the relatively heterogeneous characteristics of the human population. However, for some disorders in which there is phenotypic vulnerability, including increased stress sensitivity as a predisposition for affective disorders, it may be effective to develop rodent models by strategically selecting mouse strains based on their known characteristics (Bale, 2006). 

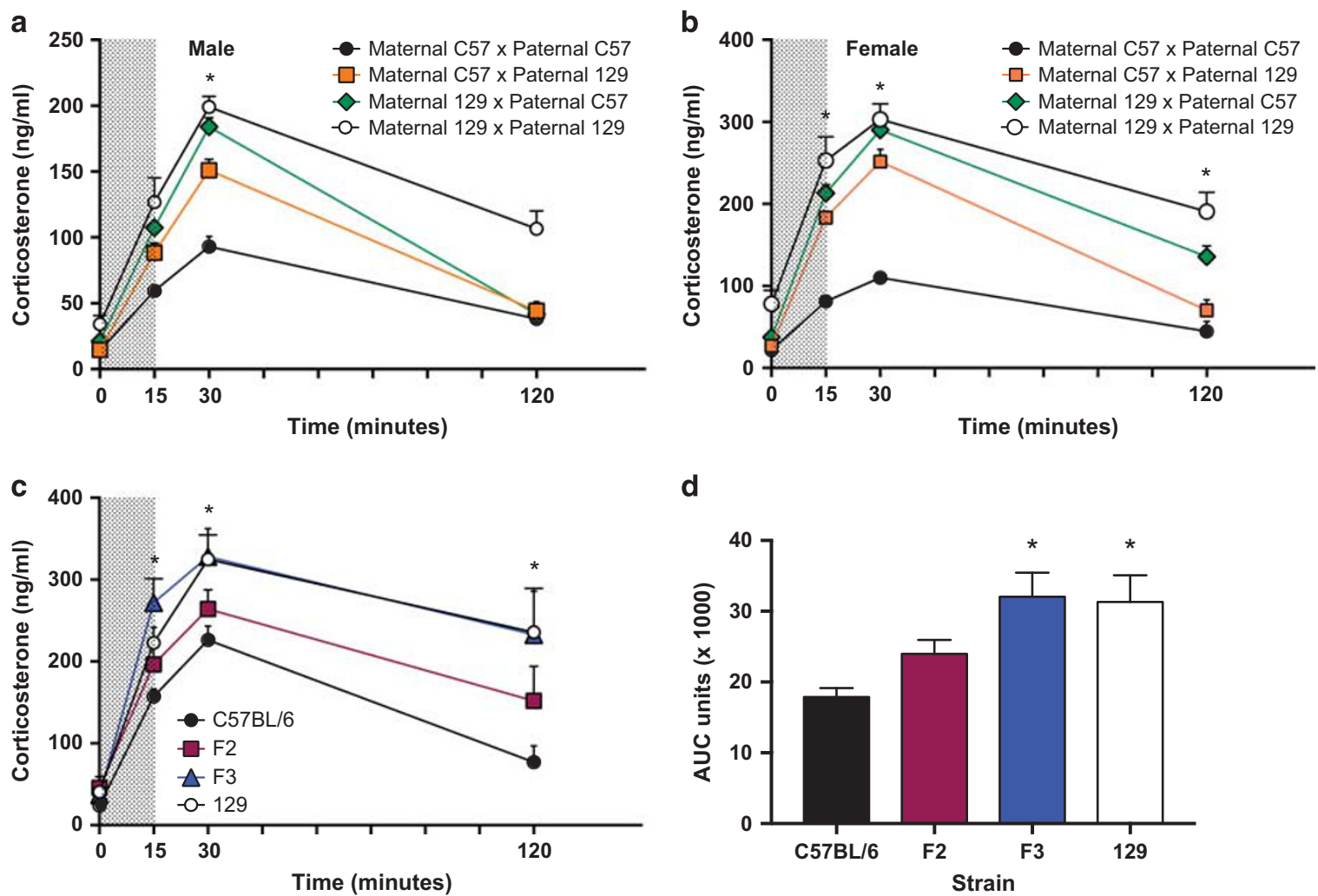

Figure I Strain-dependent differences in HPA stress axis responsiveness can be used to identify a backcrossing strategy. (a) Male and (b) female FI hybrid offspring resulting from the Maternal $129 \times$ Paternal C57 breeding scheme had greater stress reactivity than offspring from a Maternal C57 × Paternal I 29 scheme, indicated by greater levels of corticosterone at the 30-min time point in males: $F_{3,32}=32.61, p<.0001$; and a main effect on the corticosterone response curve in females: parental strain: $F_{3,34}=41.25, p<.0001$; time: $F_{3,102}=172.1, p<.000$ I; interaction: $F_{9.102}=6.82, p<.0001$. * $p<.05$ Maternal $129 \times$ Paternal C57 vs Maternal C57 × Paternal I 29 and Maternal $129 \times$ Paternal I 29 vs Maternal C57 × Paternal C57. N=6-I I/group/sex. (c) Female mice of 129 and F3 strains had significantly different corticosterone responses from C57BL/6 female mice over time (strain: $F_{3,25}=6.302, p=.0025$; time: $F_{3,75}=55.94$, $p<.000$ I; interaction: $F_{9,75}=1.28, p=.26 . N=6$-8/group. $* p<.05$ compared to C57BL/6). (d) Total corticosterone levels by area under the curve (AUC) measurements of 129 and F3 strains were significantly greater than C57BL/6 levels $\left(F_{3,25}=5.005, p=.0074\right.$. N $=6-8 /$ group. * $p<.05$ compared to $C 57 \mathrm{BL} / 6$ by post hoc analysis). The data are represented as mean \pm SEM.

More recently, to apply the mutual advantages of these known strain-specific attributes, many labs are using mixedstrain background mice (Ridder et al, 2005; Mueller and Bale, 2008; Curley et al, 2012). Researchers selectively breed two strains that have desired characteristics with the resulting offspring demonstrating a hybrid vigor (Birchler et al, 2006). For instance, in order to produce a mouse strain that is susceptible to chronic stress and still produces robust phenotypes on behavioral tasks, including cognitive performance, our lab uses C57BL/6:129 F1 hybrids for many of our studies. This method allows our lab to use a new transgenic line arriving on a C57BL/6 background for experimental testing after only one generation by crossing them with 129S1/SvImJ mice to generate the F1 hybrid. However, in the case of conditional knockouts and more complex crosses, in which two or more transgenic lines are on a C57BL/6 background, one or more lines need to be backcrossed onto a 129 strain before we can produce F1 hybrids for testing. With each mouse breeding requiring substantial time-each generation taking at least three months - we wanted to develop a biological assay as a readout to determine how many generations a C57BL/6 mouse needed to be backcrossed before producing experimental animals that would more closely resemble traits of the 129 strain.

To resolve this question, we used the robust strain differences in the hypothalamus-pituitary-adrenal (HPA) stress axis between the low stress-responding C57BL/6 and the high stress-responding 129 mouse strains as a physiological readout for inheritance of background genetics. We utilized the HPA stress axis due to its known strain differences, and as it is a robust, quantitative, and relatively non-invasive procedure that provides predictive validity for chronic stress susceptibility. There are certainly other stress measures that may also be relevant (eg, prefrontal cortex and/or amygdala reactivity) that could be explored in specific mouse strains as well (Kumar et al, 2014; Williams et al, 2015). Following each backcross, we examined the HPA stress response of the resulting offspring. The generation in which the strain differences in HPA reactivity disappeared designated the minimal number of backcrosses necessary for a mouse of C57BL/6 origin to inherit the genes underlying our desired characteristic-in this case, a heightened HPA stress axis responsiveness.

In addition, as labs interested in developmental outcomes may be backcrossing male and/or female mice of either strain, we also considered the important potential contribution of maternal $v s$ paternal genotype for inheritance of background genetics utilizing the HPA stress axis phenotype as our outcome measure. Figure $1 \mathrm{a}$ and $\mathrm{b}$ suggest that for inheritance in both male and female F1 offspring, penetrance of stress-responsive genes was greater with a maternal 
$129 \times$ paternal C57BL/6 cross, suggesting an effect of maternal/paternal imprinted genes or maternal care in the intergenerational transmission of stress phenotypes. As F1 hybrids resulting from a maternal C57BL/6× paternal 129 cross had a less reactive HPA stress response, we continued to backcross the male F1 hybrid offspring with 129 females. We exploited the known sex differences in the magnitude of the HPA stress response (ie, females having a more robust response compared to males), and focused on the female offspring from subsequent generations to produce the greatest strain differences in the HPA response curve. Surprisingly, only three backcrosses were required for the hybrid offspring to show a stress response identical to 129 mice (Figure 1c and d), suggesting F3 mice can be used to generate experimental animals for stress exposure studies.

Clearly, research requires great care in selecting the appropriate mouse strain to ensure reproducibility, face validity, and measurable outcomes across research laboratories and models. Mathematically, 10 generations are needed to ensure a strain has achieved $99.9 \%$ genetic similarity to the designated pure strain it has been crossed with (EisenerDorman et al, 2009). However, timing and mouse care expenses render this expectation extraordinarily expensive, both in terms of cage costs and research time to produce the experimental animals (ie, nearly 3 years and over $12 \mathrm{~K}$ in housing costs to generate experimental animals). We propose here that for researchers interested in stress-related research and who are confronted with backcrossing mice to a preferred strain, the HPA stress axis can be used as a robust outcome measure to designate when a mouse strain has been sufficiently backcrossed to pass on genes sufficient for stress responsivity. In our studies, three generations of backcrosses with 129 females were adequate for C57BL/6 mice to produce a 129-like HPA stress response, effectively reducing the number of necessary backcrosses to produce experimental animals by years. Following generation of experimental animals, researchers can continue backcrossing their mice with new breeders to achieve more genetic similarity with the designated pure strain, and to avoid genetic drift that may occur within their mouse colony (Casellas, 2010).

\section{FUNDING AND DISCLOSURE}

This work is funded by NIMH grants: MH108286, MH099910, and MH104184. The authors declare no conflict of interest.

\section{REFERENCES}

Anisman H, Matheson K (2005). Stress, depression, and anhedonia: caveats concerning animal models. Neurosci Biobehav $R$ 29: $525-546$.

Bale T (2006). Stress sensitivity and the development of affective disorders. Horm Behav 50: 529-533.

Birchler J, Yao H, Chudalayandi S (2006). Unraveling the genetic basis of hybrid vigor. Proc National Acad Sci 103: 12957-12958.

Brown R, Wong A (2007). The influence of visual ability on learning and memory performance in 13 strains of mice. Learn Mem 14: 134-144.

Camp M, MacPherson K, Lederle L, Graybeal C, Gaburro S, DeBrouse L et al (2012). Genetic strain differences in learned fear inhibition associated with variation in neuroendocrine, autonomic, and amygdala dendritic phenotypes. Neuropsychopharmacology 37: 1534-1547.

Casellas J (2010). Inbred mouse strains and genetic stability: a review. Animal 5: 1-7.

Curley JP, Jensen CL, Franks B, Champagne FA (2012). Variation in maternal and anxiety-like behavior associated with discrete patterns of oxytocin and vasopressin 1a receptor density in the lateral septum. Horm Behav 61: 454-461.

Ducottet C, Belzung C (2005). Correlations between behaviours in the elevated plus-maze and sensitivity to unpredictable subchronic mild stress: evidence from inbred strains of mice. Behav Brain Res 156: 153-162.

Eisener-Dorman A, Lawrence D, Bolivar V (2009). Cautionary insights on knockout mouse studies: the gene or not the gene? Brain Behav Immun 23: 318-324.

Hefner K, Whittle N, Juhasz J, Norcross M, Karlsson R-M, Saksida L et al (2008). Impaired fear extinction learning and cortico-amygdala circuit abnormalities in a common genetic mouse strain. J Neurosci 28: 8074-8085.

Holmes A, Wrenn C, Harris A, Thayer K, Crawley J (2002). Behavioral profiles of inbred strains on novel olfactory, spatial and emotional tests for reference memory in mice. Genes Brain Behav 1: 55-69.

Homanics G, Quinlan J, Firestone L (1999). Pharmacologic and behavioral responses of inbred C57BL/6J and Strain 129/SvJ Mouse lines. Pharmacol Biochem Behav 63: 21-26.

Kumar S, Hultman R, Hughes D, Michel N, Katz B, Dzirasa K (2014). Prefrontal cortex reactivity underlies trait vulnerability to chronic social defeat stress. Nat Commun 5: 4537.

Ledermann B (2000). Embryonic stem cells and gene targeting. Exp Physiol 85: 603-613.

Lucki I, Dalvi A, Mayorga A (2001). Sensitivity to the effects of pharmacologically selective antidepressants in different strains of mice. Psychopharmacology 155: 315-322.

Mogil J, Wilson S, Bon K, Lee S, Chung K, Raber P et al (1999). Heritability of nociception I: responses of 11 inbred mouse strains on 12 measures of nociception. Pain 80: 67-82.

Mozhui K, Karlsson R-M, Kash T, Ihne J, Norcross M, Patel S et al (2010). Strain differences in stress responsivity are associated with divergent amygdala gene expression and glutamate-mediated neuronal excitability. J Neurosci Official J Soc Neurosci 30: 5357-5367.

Mueller BR, Bale TL (2008). Sex-specific programming of offspring emotionality after stress early in pregnancy. $J$ Neurosci 28: 9055-9065.

Pothion S, Bizot J-C, Trovero F, Belzung C (2004). Strain differences in sucrose preference and in the consequences of unpredictable chronic mild stress. Behav Brain Res 155: 135-146.

Ridder S, Chourbaji S, Hellweg R, Urani A, Zacher C, Schmid W et al (2005). Mice with genetically altered glucocorticoid receptor expression show altered sensitivity for stress-induced depressive reactions. J Neurosci 25: 6243-6250.

Waterston RH, Lindblad-Toh K, Birney E, Rogers J, Abril JF, Agarwal P et al (2002). Initial sequencing and comparative analysis of the mouse genome. Nature 420: 520-562.

West DB, Boozer CN, Moody DL, Atkinson RL (1992). Dietary obesity in nine inbred mouse strains. Am J Physiol 262: R1025-R1032.

Williams L, Korgaonkar M, Song Y, Paton R, Eagles S, Goldstein-Piekarski A et al (2015). Amygdala reactivity to emotional faces in the prediction of general and medicationspecific responses to antidepressant treatment in the randomized iSPOT-D trial. Neuropsychopharmacology 40: 2398-2408.

Yoneyama N, Crabbe JC, Ford MM, Murillo A, Finn DA (2008). Voluntary ethanol consumption in 22 inbred mouse strains. Alcohol 42: 149-160. 